\title{
Diferenciando la complejidad: Tipologías de personas en situación de calle e intervención social de calidad
}

\author{
Differentiating complexity: Typologies of homeless \\ people and quality of social intervention
}

\section{Leticia Fuentes}

Leticia Fuentes es Trabajadora Social, Magíster Estudios Sociales y Políticos Latinoamericanos de la Universidad Alberto Hurtado. Actualmente trabaja en el Ministerio Público, Fiscalía Regional Sur. Unidad de Victimas y Testigos. Dirección postal Miguel Claro 444- Depto B-12 Providencia, Código postal: 7500993 Santiago, RM Chile. Email: Ifuentes@minpublico.cl

\begin{abstract}
Resumen
En Chile, las personas en situación de calle caracterizadas entre otros aspectos por no tener residencia y ser nómadas del espacio urbano, configuran una realidad social marcada por exclusiones de diversos sistemas funcionales, tales como el familiar, habitacional, el sistema de salud, el económico. Estas exclusiones, en su conjunto, determinan una compleja realidad social demandando intervenciones de calidad. En respuesta a tal desafío, este estudió exploró diferenciar dicha complejidad mediante tipologias construidas a partir de un sistema integrado de variables selectivas, que fueron obtenidas desde el primer catastro nacional de personas en situación de calle de nuestro pais. El resultado de las tipologías permitió diferenciar la realidad social de las personas en situación de calle, en complejidad extrema, alta y media. Este hallazgo permite concluir que a mayor complejidad de las tipologias, mayores son las exclusiones sociales que mantienen a los sujetos vulnerados de sus derechos fundamentales.
\end{abstract}

Palabras claves (Exclusión social; personas en situación de calle; intervención social de calidad)

\begin{abstract}
Chilean homeless people when characterized, among others aspects, by not having a residence and being nomadic trough the urban space, configure a social reality distinguished by exclusion from a range of functional systems, such as family, housing, health and economic system. These exclusions as a whole, determine a complex social reality demanding quality interventions. In response to this challenge, this study differentiated that complexity trough the development of typologies, built from an integrated system of selective variables that were obtained from the first National Register of Homeless People in Chile. These typologies allowed differentiating the social reality of people living on the streets, in extreme complexity, high and medium. This finding suggests that the greater complexity of typologies, the greater the social exclusion that keeps people damaged in their fundamental rights.
\end{abstract}

\section{Personas en situación de calle en sociedades funcionalmente diferenciadas}

"...Aunque todos los cristianos fueran santos, no por ese solo hecho se solucionaría el mal social. Hace falta también la técnica. Es necesario observar las cosas, criticar las ideas, razonar sobre los hechos, proponer planes y construir..." (San Alberto Hurtado. s.j.).

Observar desde la esfera de la calidad las intervenciones sociales que abordan el complejo fenómeno de las personas en situación de calle ${ }^{1}$, complejidad

\footnotetext{
1 Definidas como aquellas personas que carecen de un lugar fijo y seguro para pasar la noche, pernoctando en las calles, hospederías de beneficencia o comerciales, o que teniendo una vivienda, no logra contar con las condiciones mínimas para caracterizarla como tal. Esta categoría, surge en la Organización Hogar de Cristo, y se formaliza entre las organizaciones de la sociedad civil, y el Estado, a partir del primer Catastro Piloto en la comuna de Estación Central de Santiago el año 2003. Ministerio de Planificación y Cooperación de Chile. División Social. Habitando la Calle. Catastro Nacional de personas en situación de calle.2005. Pág. 129.
} 
dada por "la interacción de múltiples variables que determinan la situación de exclusión de los sujetos de los diversos sistemas funcionales (FEANTSA, 2001,2005, 2007; MIDEPLAN, 2005); se torna un desafío crucial si se considera que esta situación vulnera los derechos humanos de más de 7.000 chilenos (MIDEPLAN, 2005:85). A ello se suma que por primera vez en la historia de la política social chilena las personas en situación de calle comienzan a ser visibilizadas como sujetos de derechos al ser incorporadas al Sistema de Protección Social Chile Solidario. $^{2}$

Ausencia de derechos en personas en situación de calle traducida en exclusiones sociales persistentes de sistemas funcionales tales como, familiar, habitacional, salud, económico, y garantías estatales para resolverlas, configuran el complejo entorno en que se ubican las organizaciones que forman parte del sistema de cooperación cuya función es transformar la exclusión en inclusión (Mascareño, 2006:12).

En este nuevo y complejo entorno, el sistema estatal desde su rol social se acopla a dichas organizaciones, al reconocer sus trayectorias en intervención social con personas en situación de calle ${ }^{3}$ en busca de resolver este nuevo problema en su agenda de pobreza. Sin embargo, para Ossa (2005), a pesar "de los innumerables grupos y personas individuales que buscan intervenir para ayudar, el fenómeno de las personas en situación de calle no es una temática muy estudiada", de tal modo que al desconocer la complejidad del fenómeno, se desconoce también el impacto de sus intervenciones en los procesos de inclusión social de los sujetos. Siendo este el problema, urge conocer cuándo una intervención social orientada a personas en situación de calle es de calidad en sociedades funcionalmente diferenciadas.

Frente a este problema y pregunta de investigación el presente estudio propone la construcción de tipologías de personas en situación de calle que permitan diferenciar la complejidad de un fenómeno de causalidad multidimensional (Riquelme, 2005:44), donde "la exclusión social es una consecuencia, entre otras, de una sociedad cuya complejidad ha aumentado a tal punto que los modos habituales de enfrentamiento de la complejidad se hacen insuficie ntes,"(Rodríguez,2007:16) para luego, observar dichas tipologías desde matrices de análisis relacional complejidad/calidad y sus respectivas condiciones de calidad que aporten en inclusión social en los sistemas funcionales, de los cuales se encuentran excluidas las personas en situación de calle de manera persistente.

Se entenderá por Condiciones de Calidad en intervenciones sociales con personas en situación de calle la "correspondencia entre niveles de complejidad, es decir exclusión, y las condiciones para transfórmalas en inclusión". Estas condiciones son: altas, medias, umbral mínimo y bajas condiciones de calidad (Matus; Ibarra; Fuentes\& Pérez, U; 2007: 19). Además se entenderá por Condiciones Insuficientes, aquellas acciones asistencialista que omiten la promoción de la inclusión autónoma de los sujetos a los diversos sistemas funcionales; Condiciones del Umbral mínimo, acciones orientadas a responder a las necesidades fundamentales de los sujetos, (alimentación, techo y abrigo); Condiciones Medias, acciones que junto con abordar el umbral mínimo de la intervención articulan acciones orientadas a impulsar la inclusión social de los sujetos en el sistema salud, familiar, económico, habitacional; Condiciones Buenas de la intervención, junto con abordar el umbral mínimo y medio de la intervención, orienta sus acciones hacia la inclusión social autónoma de los sujetos en el sistema salud, familiar, económico, habitacional (Ibid).

Para Luhmann, "un sistema es una forma por tanto excluye un entorno, la inclusión indica entonces la cara interna de una forma cuya cara externa es la exclusión. Por consiguiente sólo es posible hablar de inclusión si hay exclusión." (Luhmann, 1998:172). Así planteado, la dialéctica exclusión/inclusión en personas en situación de calle adquiere un carácter relacional, entre los que están dentro (incluidos) y los que están fuera (excluidos) (Barros; de los Ríos \& Torche; 1996:95). Aquí "la noción de inclusión no puede significar que en los sistemas sociales estén

2 Chile. Ministerio de Planificación y Cooperación. Ley 19.949. Sistema de Protección Social, Chile Solidario. Julio 2006. En http://www. bcn.cl/lc/bleyes. Visitada 6 Noviembre de 2007.

3 Hogar de Cristo, Nuestra Casa, Moviliza, Cristo en la calle, Kairos, Caleta Sur, Don Bosco, entre otras. Estas organizaciones pertenecen a la Región Metropolitana. En las regiones V y VIII, regiones en que se encuentra en proceso de implementación la propuesta piloto de integración, las organizaciones refieren principalmente a programas de la Fundación Hogar de Cristo y municipios. Ministerio de Planificación y Cooperación. Secretaría Ejecutiva Chile Solidario. (2007). Listado de instituciones ejecutoras, "Programa de apoyo a la integración social de personas en situación de calle". Estrategia de intervención fase piloto del programa. 
contenidas todas aquellas operaciones que dan continuidad a la vida o que reproducen la conciencia" (Luhmann; Op.Cit); con ello se resuelve la tentación de pensar que la integración y mejoramiento de la calidad de vida de los sujetos pasaría por incluirlos en todos los sistemas de la sociedad.

Comprender el fenómeno en estudio en este escenario funcional y diferenciado implica comprender que los individuos sin hogar presentan una reconocida ruptura de reciprocidad, en contraparte, la inclusión permanece vinculada a la vida sedentaria, exigiendo de los sujetos "interacción permanente y creadora de expectativas" (Ibid); es decir, sujetos activos en cuanto sus procesos de inclusión.

En este escenario, la solución de los problemas sociales implica más especialización. De esta forma, la inclusión social no se resuelve desde un poder central o desde una organización total, pierde su carácter adscrito y se transforma en una tarea que se desarrolla mediante mecanismos sociales (competitivos) de selección (Luhmann,1992). Estos mecanismos hacen alusión a las carreras personales de los sujetos, sus capacidades, su capital social, su nivel educacional, de tal modo que una sociedad funcionalmente diferenciada será o debe pretender ser una sociedad de iguales donde los sistemas funcionales son desiguales, pero sus correspondientes entorno deben tratarse como entornos de iguales, así conceptualizada la exclusión/inclusión se torna temporal y limitada (Ibid) a los concretos sistemas de la sociedad.

Para Razeto (1993:38) el nivel de vida y las oportunidades que nos ofrecen en la vida dependen de las donaciones que hayamos recibido en la infancia y juventud. Las personas en situación de calle reflejan los enunciados de Razeto al presentar déficit en cuanto a sus carreras personales, situándose en clara desventaja frente a otros que han contado con oportunidades para acceder a sistemas educativos, contar con familias funcionales, trabajos formales, salud oportuna y con escasas estigmatizaciones que deterioren su identidad. Asimismo, Sen (1995:32), hablando de desigualdad señala que "comenzamos a vivir con diferentes dotes de riquezas y compromisos heredados. Habitamos en diferentes ambientes naturales unos más hostiles que otros. Las sociedades de las que formamos parte nos ofrecen diversas oportunidades de lo que podemos o no podemos hacer".
Frente a esta pluralidad de variables que disminuyen las "carreras personales" de las personas en situación de calle, las asimetrías son significativas con el resto de la población, no sólo en relación a una variable focal como le llamaría Sen, sino que presentan desigualdades multivariadas, siendo estas desigualdades y exclusiones las que se tornan persistentes, alejándolas de exclusiones/inclusiones temporales y limitadas propias de sociedades funcionalmente diferenciadas, ${ }^{4}$ configurando el complejo entorno en que se encuentran.

\section{Principales exclusiones presentes en personas en situación de calle}

\section{Sistema familiar: Derecho al afecto, al reconocimiento}

Es Mascareño (2005) quien señala que una de las exclusiones fundamentales presente en personas en situación de calle es la imposibilidad de comunicarse con otros sujetos de la sociedad que les permita la confirmación de su propia existencia. Esta dimensión sociocultural de la exclusión dificulta la incorporación de las personas al quehacer y a la dinámica de la sociedad (Barros; de los Ríos \& Torche; 1996). Bravo (2004), identifica tres factores de complejidad, un factor de dominio estructural relacionado con la dimensión económica de la exclusión, haciendo referencia a la exclusión del mercado laboral; un factor de dominio social, manifestado en la exclusión de las redes primarias (familia) como secundaria (redes de apoyo comunitario) y un factor de dominio personal o vivencial del sujeto, que presenta una ruptura de la comunicación y la erosión de virtudes tales como la confianza básica, identidad, la reciprocidad y la solidaridad.

La ausencia sistemática de intercambio con sistemas funcionales conlleva a las personas en situación de calle ha permanecer al margen de posibilidades mínimas de inclusión, lo cual trae aparejado consecuencias en cadena, impulsos sistémicos que exigen rendimiento en otros sistemas. En palabras de Luhmann "las familias que viven en calle y no tienen dirección fija no pueden matricular a sus hijos en la escuela. O quien no tiene documentación personal queda excluido de las prestaciones sociales, no puede ser lector ni casarse legalmente."(Luhmann, citado por Mascareño, 2006:6). Repercusiones en

4 Al ser consultadas las personas en situación de calle el motivo por el que se encuentran en esta situación, hablan de problemas familiares, problemas económicos, falta de casa-hogar. En MINISTERIO de Planificación y cooperación de Chile, División Social. (2005). Habitando la Calle. Catastro nacional de personas en situación de calle. Santiago, Chile. Pág.97. 


\section{EXCLUSIONES PRESENTES EN PERSONAS EN SITUACIÓN DE CALLE Y SU INTERRELACIÓN}

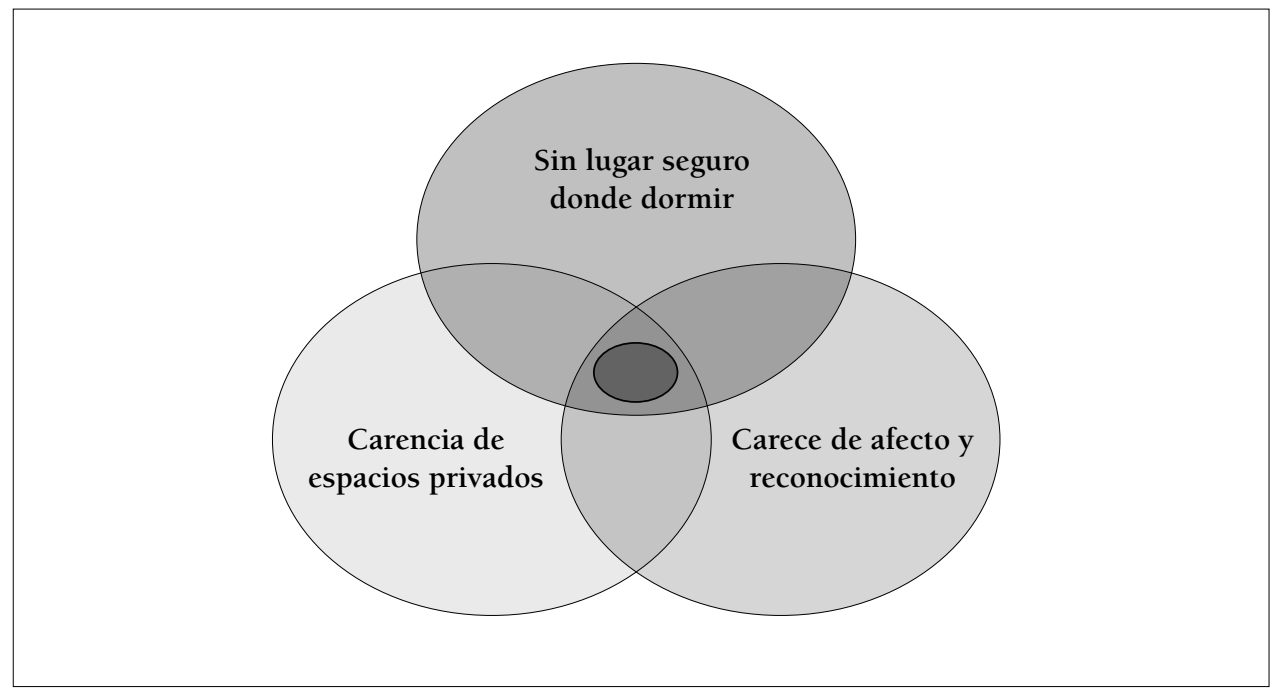

Fuente: Adaptación del modelo presentado por Meert et. al. (2005:4)

cadena son señales de que el fenómeno no es lineal, causa-efecto, sino complejo, recursivo. Así como las exclusiones repercuten en cadena en otros sistemas, cualquier intento de intervención positiva presentará resonancia en otros dominios vinculados a la condición de calle. La figura siguiente grafica lo señalado.

\section{Sistema habitacional: Derecho a la privacidad}

Lograr contar con alojamiento adecuado y seguro es una condición previa al ejercicio de otros derechos fundamentales. "Ser una persona sin hogar, sin acceso a alojamiento digno es probablemente la manifestación más seria de la exclusión social"
(FEANTSA, 2001:22) al dificultar el despliegue de potencial personal como miembro activo de una sociedad. En este sentido, para FEANTSA el acceso al alojamiento estable, es la clave para lograr inclusión social, identificando la siguiente tipología denominada ETHOS (Ibid).

En Chile, tipologías referentes a la situación habitacional de personas en situación de calle se desprenden de la definición utilizada en el catastro nacional (MIDEPLAN: 2005:129).

1. Personas en situación de calle que se encuentren pernoctando en lugares públicos o privados, sin contar con infraestructura tal que pueda ser ca-

TIPOLOGÍA EUROPEA DE PERSONAS SIN HOGAR Y EXCLUSIÓN RESIDENCIAL: ETHOS

\begin{tabular}{|l|l|}
\hline Categoría conceptual & Definición \\
\hline Sin techo & $\begin{array}{l}\text { - Vivir en un espacio público sin domicilio } \\
\text { - Pernoctar en albergue y pasar el resto del día en un espacio público }\end{array}$ \\
\hline Sin vivienda & $\begin{array}{l}\text { - Estancia en centros de servicios o refugios. } \\
\text { - Vivir en estancias para mujeres } \\
\text { - Vivir en instituciones }\end{array}$ \\
\hline Vivienda insegura & $\begin{array}{l}\text { - Vivir en vivienda sin título legal } \\
\text { - Vivir temporalmente con familiares } \\
\end{array}$ \\
\hline Vivienda inadecuada & $\begin{array}{l}\text { - Vivir en una estructura temporal e inadecuada } \\
\text { - Vivienda no adecuada según la legislación estatal } \\
\text { - Vivir en vivienda masificada }\end{array}$ \\
\hline
\end{tabular}

Fuente: Meert et.al. (2005:4) 
racterizada como vivienda aunque la misma sea precaria.

2. Persona en situación de calle que por carecer con alojamiento fijo regular y adecuado para pasar la noche encuentran residencia nocturna pagando o no por este servicio, en alojamiento dirigido por entidades públicas, privadas o particulares y que brindan albergue temporal.

3. Personas en situación de calle que por encontrarse sin hogar y sin apoyo de familiares u otros significativos, dependen de programas sociales que ofrecen residencia permanente por periodos importantes, con apoyo biosicosocial.

Ambas tipologías (FEANTSA y MIDEPLAN) presentan dos distinciones en común, las que se encuentran residiendo en calle, y aquellas que se encuentran pernoctando en residencias de manera regular, hospederías gratuitas o comerciales.

A su vez, nuestro país incorpora a la categoría de personas en situación de calle a sujetos que se encuentran de manera permanente residiendo en hogares o programas residenciales, como es el caso de los adultos mayores. Por considerar que al ser egresados de dichos programas volverían a pernoctar en espacios públicos. De acuerdo a ello, las personas que viven en calle "comprendidas como aquellas que viven en sitios eriazos, vía pública y caleta, tienden a estar bastante más desprotegidas que las personas que viven en hospederías, puesto que éstas tienen algún tipo de asistencia, apoyo biosicosocial o simplemente cuentan con una residencia (lugar donde llegar) que cumple a lo menos con las condiciones de resguardo básico."(Ibid).

Los datos recogidos por el catastro nacional dan cuenta que el $52 \%$ de las personas catastradas duermen habitualmente en hospederías, mientras que un $40.6 \%$ se encontraba pernoctando en la calle y un $15.4 \%$ restante pernoctaban en un lugar distinto a los mencionados (Op. Cit:87).

\section{Sistema de salud: Derecho a contar con protección de salud}

Junto al derecho a la vivienda, las personas en situación de calle presentan exclusiones sociales relativas al sistema de salud siendo un factor vital para la inclusión social (FEANTSA, 2004:4). La Organización Mundial de la Salud, establece que la salud "es un estado de completo bienestar físico, mental y social y no meramente la ausencia de enfermedad o debilidad". En Francia, informes sobre el tema señalan que "no hay enfermedades específicas de la situación de los sin techo, más bien la situación sanitaria empeora en relación directa con su situación en su conjunto."(Op. Cit:6). Nuestro país no cuenta con estudios específicos respecto al tema que den cuenta de las principales enfermedades que les aquejan, más bien la relación establecida es desde salas de urgencia donde en algunos casos han sido observados como "casos sociales" al carecer de cédulas de identificación, ausencia de redes primarias activas en la recepción de entrega de información.

Para FEANTSA hay muchos factores que puede convertir a una persona en situación de calle, siendo la salud un factor de ellos. Un sujeto en esta situación presentará a menudo tres o más trastornos, tales como, problemas de salud mental, abuso de sustancias, trastornos de la personalidad, trastornos depresivos, dificultades de aprendizajes, discapacidad, problemas físicos de salud, vulnerabilidad a causa de la edad (Op. Cit: 7). Estas necesidades múltiples pueden complicarse con experiencias negativas con el sistema sanitario. O Connell (2004:3) destaca otras enfermedades que las personas en situación de calle se encuentran vulnerables de adquirir por los estilos de vida, como enfermedades infecciosas, alergias, pediculosis, enfermedades de transmisión sexual, diversos tipos de hepatitis, entre otras.

El catastro nacional muestra que un $13.1 \%$ de las personas entrevistadas le atribuyen a la variable salud la principal causa de su situación actual, un $16.7 \%$ sindica estar en esta situación por consumo problemático de alcohol y un $7.1 \%$ lo relaciona a su consumo de drogas (MIDEPLAN, 2005:97). A lo anterior se agrega, como indicador de complejidad que estos sujetos presentan menos acceso a los recursos sanitarios básicos y al sistema de salud en su conjunto, es por ello que enferman y mueren con mayor frecuencia que aquellos grupos que ocupan posiciones sociales más privilegiadas.

\section{Sistema económico: Derecho a trabajar}

Las personas en situación de calle al presentar exclusiones de vivienda y salud, se sitúan en desventaja de oportunidades para encontrar trabajo. "Para que una persona pueda acceder al mercado laboral más o menos normalizado, se hace necesario que la persona tenga las necesidades básicas de vivienda, y comida satisfechas y hábitos de higiene y puntualidad. Se hace necesario llegar a estos mínimos para iniciar un proceso de incorporación y búsqueda de empleo" (FEANTSA, 2007:6)

Frente a estas dificultades para insertarse el mundo formal de trabajo, las personas en situación de 
calle desarrollan estrategias de sobrevivencia. Bravo (2004:34), las caracteriza como irregulares, intermitentes, ocasionales e informales.

El catastro nacional da cuenta de la creatividad de los sujetos en la búsqueda ampliada de estrategias de sobrevivencia; vendedor ambulante, cuidador de autos, obrero, macheteo, cargador, cartonero, temporero, jardinería, empleado, recolector, artista callejero, artesanía, mecánico, júnior, servicios domésticos entre otras (MIDEPLAN, 2005:125). Estas ocupaciones informales permiten observar que los sujetos presentan disposición positiva al trabajo a pesar de las condiciones adversas en las que viven. En este sentido, la principal fuente de ingreso de las personas en situación de calle proviene del trabajo $27.2 \%$, donde el macheteo o limosna corresponde el $17.0 \%$.

Se observa que las mujeres se encuentran en desventaja al considerar que los ingresos de los hombres duplican al de las mujeres. Otras fuentes de ingreso, que rescata el informe del catastro, son la jubilación, correspondiendo a un 5.1\%, así como también lo son las transferencias económicas del Estado, alcanzando a un 30\%, consistente en PASIS vejez o invalidez.

En síntesis, la heterogeneidad y cronicidad de exclusiones a las que se sitúan las personas en situación de calle, reclaman intervenciones diferenciadas en sociedades modernas. Donde las condiciones de calidad varían según las exclusiones que presenten los sujetos, tiempo de permanencia en calle, según sexo o edad, o frente a aquellos que requieren salud y otros trabajo, los que sufren discapacidad física de aquellos que los aqueja un trastorno de salud mental, los que duermen en calle de aquellos que lo hacen en hospederías, o frente aquellos que mantienen contacto con el sistema familiar, de aquellos que no lo hacen en años o no lo desean; o de aquellos que desean permanecer en calle persistentemente de aquellos que anhelan inclusión. Sen, hablando de desigualdad expresa: "que considerar a todos por igual puede resultar en que se dé un trato desigual a aquellos que se encuentran en una posición desfavorable" (1995:14).

\section{Complejidad y condiciones de calidad: Una relación necesaria en intervenciones sociales con personas en situación de calle}

Para Matus et. al. (2007:17), comprender la complejidad del sistema/entorno es fundamental al pensar en calidad, "la calidad es un concepto que le sigue inmediatamente al nivel e intensidad de la complejidad". Así formulado, es necesario conocer qué intervención social requieren los sujetos de acuerdo a diversas tipologías de complejidad.

Esta necesidad de diferenciación es reconocida desde el Ministerio de Planificación, donde a partir de la Propuesta Piloto de Integración Social orientada a Personas en Situación de Calle buscan como producto "un conjunto de estrategias de intervención diferenciales para entregar protección social a personas viviendo en situación de calle" (MIDEPLAN, 2006:3).

En sociedades diferenciadas, el llamado a resolver el problema de la exclusión es el sistema de cooperación cuya función se expresa en la transformación de la exclusión en inclusión y se materializan en sus organizaciones. El sistema de la cooperación ha logrado decantar un medio de comunicación simbólicamente generalizado, el medio de la colaboración, una suficiente abstracta formula de contingencia como la solidaridad y acoplamientos estructurales crecientemente densos especialmente con el sistema económico, político y jurídico" (Mascareño, 2006:2).

En este sistema, la exclusión social, es la vulneración de derechos humanos cuyo pilar son los derechos sociales. Estos derechos adquieren un carácter cosmopolita al trascender la pertenencia política del individuo (Op. Cit:8).

Para Vicente (2006:20-21), el recibir atención deficitaria en el sistema de salud, el ser discriminado frente a la búsqueda de empleo, y frente otras situaciones excluyentes, hablan de vulneración de derechos sociales. El salto que se requiere desde el Estado garante de tales derechos, es avanzar desde una declaración formal, al reconocimiento jurídico auténtico, que les asegure el ejercicio de derechos mediante garantías jurídicas adecuadas.

En la misma línea, se ha señalado que solo el reconocimiento integral de los derechos económicos, sociales, y culturales puede asegurar la existencia real de ellos. De este modo, la exclusión social vista desde la vulneración de derechos sociales en sociedades diferenciadas alcanza su máximo exponente en las personas sin hogar (CARITAS ESPAÑOLA, 2006:2). 


\section{CRITERIOS FUNCIONALISTAS SELECCIONADOS QUE BUSCAN APORTAR A UNA INTERVENCIÓN SOCIAL DE CALIDAD}

\begin{tabular}{|c|c|c|}
\hline Criterios & Indicadores & Interpretación \\
\hline $\begin{array}{l}\text { Enfoque de } \\
\text { intervención de } \\
\text { orientación } \\
\text { sistémica contextual }\end{array}$ & $\begin{array}{l}\text { - Reconoce contexto sociocultural en que se } \\
\text { ubican los sujetos } \\
\text { - Reconoce solo la individualidad de los sujetos } \\
\text { - No amplía posibilidades de inclusión }\end{array}$ & $\begin{array}{l}\text { A mayor conocimiento del carácter } \\
\text { contextual de la intervención, ma- } \\
\text { yor calidad de la intervención social }\end{array}$ \\
\hline $\begin{array}{l}\text { Diferenciación } \\
\text { de complejidad }\end{array}$ & $\begin{array}{l}\text { - Diferencia complejidad a partir de un sistema } \\
\text { integrado de variables } \\
\text { - Diferencia complejidad a partir de variables } \\
\text { sexo, edad, dónde duerme } \\
\text { - Diferencia complejidad a partir de una } \\
\text { variable focal } \\
\text { - No diferencia complejidad "todos por igual" }\end{array}$ & $\begin{array}{l}\text { A mayor diferenciación de la } \\
\text { intervención según tipologías de } \\
\text { complejidad, mayor calidad de la } \\
\text { intervención }\end{array}$ \\
\hline $\begin{array}{l}\text { Acoplamiento } \\
\text { estructural }\end{array}$ & $\begin{array}{l}\text { - Se acopla a sistemas funcionales y sistema } \\
\text { estatal } \\
\text { - Se acopla a sistemas a los cuales los sujetos } \\
\text { requieren incluirse } \\
\text { - Se acopla a sistemas que discriminan a } \\
\text { personas en situación de calle } \\
\text { - No se acopla a sistemas funcionales }\end{array}$ & $\begin{array}{l}\text { A mayor acoplamiento estructural } \\
\text { con sistemas funcionales y entorno, } \\
\text { mayor calidad de la intervención } \\
\text { social }\end{array}$ \\
\hline $\begin{array}{l}\text { Sustentabilidad } \\
\text { de la intervención }\end{array}$ & $\begin{array}{l}\text { - Sujetos se incluyen a sistemas y egresan } \\
\text { de los programas de intervención } \\
\text { - Sujetos egresan de programas, } \\
\text { e ingresan nuevamente } \\
\text { - Intervención desarrolla competencia } \\
\text { en sujetos } \\
\text { - Sujetos se mantienen dependientes } \\
\text { a los programas de intervención }\end{array}$ & $\begin{array}{l}\text { A mayor ampliación de autonomía } \\
\text { de inclusión, mayor sustentabilidad } \\
\text { y calidad de la intervención social }\end{array}$ \\
\hline
\end{tabular}

Fuente: Elaboración propia.

\section{Intervención de orientación sistémica contextual}

La intervención social sólo es posible como orientación sistémica contextual, entendida como una "invitación e incentivo a la autorregulación de sistemas (sociales y psíquicos) autónomos, operativamente clausurados y cognitivamente abiertos a su entorno sobre la base de sus propias distinciones"(Mascareño, 1996). De tal modo que los interesados en resolver este problema, organizaciones, Estado, requieren desplegar intervenciones sociales cuyos objetivos se orienten a regular los obstáculos que impiden a las personas en situación de calle el acceso efectivo de sus derechos como ciudadanos, partiendo del reconocimiento de su individualidad, donde la base del "el éxito dependerá de la incorporación que el sistema a regular haga de las distinciones propuestas. Es decir, el éxito final regulativo no está en el regulador ni en su estrategia, sino que en la incorporación que el propio sistema que es transformado hace de la oferta de regulación" (Mascareño, 2007) así, la intervención social bus- cará captar las especificidades de las personas en situación de calle, y desde su lenguaje, necesidades, sueños, expectativas, ofrecer las distinciones significativas a los sujetos. Cuando ello se logre, serán las propias personas en situación de calle que reconocerán la propiedad y la conveniencia de adoptar las alternativas ofrecidas.

La intervención social no se impone, "no hay coacción ni compulsión" (Ibid), por tanto, un indicador de eficiencia de la estrategia de intervención en personas en situación de calle, será la autoaceptación que se produce, evidencia que los sujetos aceptan lo ofrecido. Lo que se busca es una "intervención social que sea una oferta real a partir de los cuales los sujetos tengan posibilidades contingentes de elegir formas específicas de fomento de autonomía."(Matus, 2004:15).

Captar la especificidad de las personas en situación de calle, y de los sistemas en los que requieren incluirse, es captar "el carácter contextual de la intervención, es una precondición sistémica” (Mas- 
careño, 2007), donde la clausura del sistema solo posibilita actuar por el contexto, que hace referencia a un marco general de condiciones que orienta las formas concretas de operar,"la intervención social se constituye como una forma de ver qué funda un hacer peculiar. Allí, existe un vínculo que no puede ser roto, porque no hay buenas acciones sociales sin una comprensión compleja de lo social" (Matus, 2004:12).

\section{Diferenciación de complejidad}

Al reconocer que el fenómeno en estudio no responde a un fenómeno de causalidad simple (MIDEPLAN, 2005:89), muy por el contrario la situación de exclusión social responde la interacción de múltiples variables (Gerber, 2006:69), se torna fundamental diferenciar la complejidad, y con ello evitar anular la individualidad de los sujetos, sus particularidades, sueños, y libertades personales que varían de acuerdo a sus historias de vida.

\section{Acoplamiento estructural}

Los sujetos presentan baja resonancia hacia sistemas funcionales que presenten obstáculos en su desarrollo, por tanto, las organizaciones requieren asumir condiciones de calidad que incorporen este criterio sistémico en la intervención con personas en situación de calle, toda vez, que "los acoplamientos estructurales reducen la complejidad entre sistemas y el entorno en la medida que seleccionan, privilegian determinadas fuentes de irritaciones respecto de otras. Los sistemas acoplados entre si provocan perturbaciones recíprocas, las cuales en los sistemas respectivos, pueden ser percibidas como opor- tunidades para la construcción de estructuras de expectativas" (Mascareño, 1999:11).

\section{Sustentabilidad de la intervención}

Este criterio funcionalista se traduce en la posibilidad de los sujetos de transmitir a otros posibilidades de inclusión, sustentabilidad de la intervención en el marco de la orientación sistémica contextual, donde EGO (personas en situación de calle) al recibir libertad de inclusión de ALTER (interventor como organizaciones, política social), la trasmite mediante acciones similares a otros indeterminados que vivencien situaciones de exclusión (Arnold; Thumala, 2006:15) con ello, se estará aportando a la sustentabilidad de la intervención recibida.

\section{Metodología}

Estudio de carácter exploratorio con presencia de elementos descriptivos (Hernández; Fernández, 2000:58) asociado a la construcción de tipologías. Lo exploratorio responde a los escasos estudios en el tema, y las obras existentes no recogen la complejidad de la realidad y su relación con la calidad de la intervención. Sin embargo, el fenómeno ha ido adquiriendo relevancia en nuestro país a partir del catastro nacional, y la visibilización que el sistema estatal ha realizado al incorporar a las personas en situación de calle al discurso público.

\section{Selección de las variables}

Realizada a partir del resultado diagnóstico del fenómeno y de la disponibilidad de variables presentes en la base de datos del catastro nacional de personas en situación de calle. 
VARIABLES SELECCIONADAS PARA LA ELABORACIÓN DE TIPOLOGÍAS

\begin{tabular}{|c|c|c|}
\hline Variable & Indicadores & Interpretación \\
\hline V1: Sexo & $\begin{array}{l}\text { Masculino } \\
\text { Femenino }\end{array}$ & $\begin{array}{l}\text { A mayor personas de sexo femenino } \\
\text { mayor complejidad de la tipología }\end{array}$ \\
\hline V2: Edad & $\begin{array}{l}59 \text { años y menos } \\
60 \text { años y mas }\end{array}$ & $\begin{array}{l}\text { A mayor asociación de menores de } 59 \\
\text { años con lugar donde duerme y perma- } \\
\text { nencia en calle, mayor complejidad de } \\
\text { la tipología }\end{array}$ \\
\hline $\begin{array}{l}\text { V3: Lugar donde } \\
\text { duerme habitualmente }\end{array}$ & $\begin{array}{l}\text { Calle } \\
\text { Hospedería }\end{array}$ & $\begin{array}{l}\text { A mayor personas que duermen en } \\
\text { calle, mayor complejidad de la tipología }\end{array}$ \\
\hline $\begin{array}{l}\text { V4: Tiempo que lleva en } \\
\text { situación de calle }\end{array}$ & $\begin{array}{l}\text { Menos de } 1 \text { año } \\
\text { Entre } 1 \text { y } 5 \text { años } \\
\text { Mas de } 5 \text { años }\end{array}$ & $\begin{array}{l}\text { A mayor número de años en calle, ma- } \\
\text { yor complejidad de la tipología }\end{array}$ \\
\hline $\begin{array}{l}\text { V5: Tiempo que se } \\
\text { comunicó con la familia }\end{array}$ & $\begin{array}{l}1 \text { año o menos } \\
1 \text { y } 5 \text { años } \\
\text { Mas de } 5 \text { años } \\
\text { No tiene familia/no se acuerda }\end{array}$ & $\begin{array}{l}\text { A mayor número de años sin co- } \\
\text { municación con la familia mayor } \\
\text { complejidad de la tipología }\end{array}$ \\
\hline V6: Con quién vive & $\begin{array}{l}\text { No vive solo } \\
\text { Si vive solo }\end{array}$ & $\begin{array}{l}\text { A mayor personas que viven solas, } \\
\text { mayor complejidad de la tipología }\end{array}$ \\
\hline $\begin{array}{l}\text { V7: Motivo por el que vive en } \\
\text { calle: Problema con la familia }\end{array}$ & $\begin{array}{l}\text { Si problemas con la familia } \\
\text { No problemas con la familia }\end{array}$ & $\begin{array}{l}\text { A mayor motivos asociados a problema } \\
\text { con la familia, mayor complejidad de la } \\
\text { tipología }\end{array}$ \\
\hline $\begin{array}{l}\text { V8: Motivo por el que vive } \\
\text { en calle: No con vivienda }\end{array}$ & $\begin{array}{l}\text { Si tiene } \\
\text { No tiene }\end{array}$ & $\begin{array}{l}\text { A mayor porcentaje de sujetos que } \\
\text { declaran no tener casa, mayor comple- } \\
\text { jidad de la tipología }\end{array}$ \\
\hline $\begin{array}{l}\text { V9: Motivo por el que vive } \\
\text { en calle: Problema de salud }\end{array}$ & $\begin{array}{l}\text { Si problemas de salud } \\
\text { No problemas de salud }\end{array}$ & $\begin{array}{l}\text { A mayor problemas de salud, mayor } \\
\text { complejidad de la tipología }\end{array}$ \\
\hline $\begin{array}{l}\text { V10: Motivo por el que vive } \\
\text { en calle: Problemas drogas }\end{array}$ & $\begin{array}{l}\text { Si problemas de droga } \\
\text { No problemas de droga }\end{array}$ & $\begin{array}{l}\text { A mayor problemas de drogas, mayor } \\
\text { complejidad de la tipología }\end{array}$ \\
\hline $\begin{array}{l}\text { V11: Motivo por el que vive } \\
\text { en calle :Problemas de alcohol }\end{array}$ & $\begin{array}{l}\text { Si problemas de alcohol } \\
\text { No problemas de alcohol }\end{array}$ & $\begin{array}{l}\text { A mayor problemas de alcohol, mayor } \\
\text { complejidad de la tipología }\end{array}$ \\
\hline $\begin{array}{l}\text { V12: Motivo por el que vive } \\
\text { en calle :Problema económico }\end{array}$ & $\begin{array}{l}\text { Si problema económico } \\
\text { No problema económico }\end{array}$ & $\begin{array}{l}\text { A mayor problemas económicos, mayor } \\
\text { complejidad de la tipología }\end{array}$ \\
\hline $\begin{array}{l}\text { V13: Motivo por el que vive } \\
\text { en calle : Falta de Trabajo }\end{array}$ & $\begin{array}{l}\text { Si trabajo } \\
\text { No trabajo }\end{array}$ & $\begin{array}{l}\text { A mayor personas sin trabajo, mayor } \\
\text { complejidad de la tipología }\end{array}$ \\
\hline V14:Macheo /limosna & $\begin{array}{l}\text { Si macheteo /limosna } \\
\text { No macheteo/ limosna }\end{array}$ & $\begin{array}{l}\text { A mayor personas macheteo/ limosna, } \\
\text { mayor complejidad de la tipología }\end{array}$ \\
\hline V15: Recibe dinero & $\begin{array}{l}\text { Si dinero } \\
\text { No dinero }\end{array}$ & $\begin{array}{l}\text { A mayor personas que no reciban dine- } \\
\text { ro, mayor complejidad de la tipología }\end{array}$ \\
\hline $\begin{array}{l}\text { V16: Aspectos positivos y } \\
\text { negativos de vivir en calle: }\end{array}$ & $\begin{array}{l}\text { Si riesgo / No riesgo } \\
\text { Si desesperanza / No desespe- } \\
\text { ranza } \\
\text { Si malas condiciones de vida } \\
\text { /No malas condiciones de vida } \\
\text { Si hambre / No hambre }\end{array}$ & $\begin{array}{l}\text { A mayor aspectos negativos de vivir en } \\
\text { calle, mayor complejidad de la tipología }\end{array}$ \\
\hline
\end{tabular}

Fuente: Elaboración propia.

Valores de complejidad asignados a los indicadores de las variables: Al tratarse de 16 variables los valores totales de complejidad son; Complejidad extrema, 49 a 64 puntos; Complejidad alta, 33 a 48 puntos; Complejidad media, 17 a 32 puntos; Complejidad baja, 1 a 16 puntos. 


\section{Elaboración de tipologías}

Se utilizó la base de datos del catastro nacional de personas en situación de calle realizado en 80 comunas de más de 40.000 habitantes del país (MIDEPLAN, 2005). Comprendiendo un total de 6.942 sujetos, con un número significativos de casos perdidos. Respecto a estos casos, Gerber postula que "mientras mayor es el porcentaje de datos perdidos, mayor es el nivel de complejidad"(Gerber, 2006:97), siendo atribuibles a múltiples variables de complejidad en que se encuentran los sujetos al momento de ser entrevistado.

Se realiza un análisis previo de las variables en términos teóricos y empíricos, resultando esencial para evaluar la pertinencia de las tipologías ha construir. Para ello, se aplicaron pruebas de Chi Cuadrado a distintas combinaciones de variables, y se observaron las frecuencias, al constatar que la significación de la prueba fue menor a 0,05 y el Chi Cuadrado fue alto, se confirmó que las variables seleccionadas para la construcción de tipologías se encontraban asociadas.

El procedimiento estadístico seleccionado fue Two Step Cluster, al considerar el análisis de tipologías de tipo categórico (cualitativas) y ordinal, siendo un procedimiento diseñado para analizar la complejidad (Op. Cit: 69), mediante análisis multivariable. Asimismo, es una técnica que se corresponde con el carácter de la investigación, en tanto se utiliza con fines exploratorios para buscar agrupaciones naturales en los datos (Cead' Ancona, 2002:229).

En consecuencia, hay que destacar que al realizar los análisis de tipologías se dejó al procedimiento iterar hasta encontrar la mejor solución en los datos sin especificar a priori el número de grupos que se quería formar. En cuanto a la elaboración de matrices de análisis relacional, complejidad/calidad, se adaptó "la matriz de análisis relacional de complejidad" presentada por Matus; Ibarra; Fuentes \& Pérez, 2007:19).

La matriz anteriormente presentada permitirá, "componer en forma general o descomponer por áreas" (Ibid), posibilitando la observación de tipologías de complejidad tanto por sistemas, (familia, salud, economía, habitacional) como por tipologías generales, favoreciendo en ambos casos orientar intervenciones de acuerdo a tipologías de complejidad y sus respectivas condiciones de calidad.

\section{CONDICIONES PARA INTERVENCIONES SOCIALES DE CALIDAD}

\begin{tabular}{|c|l|l|l|l|l|}
\hline & Extrema & $\begin{array}{l}\text { Buenas } \\
\text { Tipologías de personas en } \\
\text { situación de calle de } \\
\text { complejidad extrema }\end{array}$ & & $\begin{array}{c}\text { Umbral } \\
\text { mínimo }\end{array}$ & Nivel insuficiente \\
\hline \multirow{2}{*}{$\begin{array}{c}\text { Complejidad } \\
\text { de } \\
\text { tipologías }\end{array}$} & Alta & & & & \\
\cline { 2 - 6 } & Bedia & & & & \\
\cline { 2 - 6 } & Baja & & & $\begin{array}{l}\text { Tipologías de personas } \\
\text { en situación de calle de } \\
\text { complejidad baja }\end{array}$ \\
\hline
\end{tabular}

\section{Resultados}

TIPOLOGÍAS DE COMPLEJIDAD

\begin{tabular}{|c|c|c|}
\hline Tipologías & $\mathbf{N}$ & $\%$ \\
\hline 1 & 1.037 & $28.2 \%$ \\
\hline 2 & 896 & $24.4 \%$ \\
\hline 3 & 922 & $25,1 \%$ \\
\hline 4 & 822 & $22.4 \%$ \\
\hline Combinaciones & 3677 & $100 \%$ \\
\hline Casos excluidos & 3265 & \\
\hline Total & 6942 & \\
\hline
\end{tabular}

Fuente: Elaboración propia. 
VALORES DE COMPLEJIDAD ASIGNADOS A VARIABLES SELECCIONADAS POR TIPOLOGÍAS

\begin{tabular}{|c|c|c|c|c|c|c|c|c|c|c|c|c|c|c|c|c|c|}
\hline $\begin{array}{c}\text { Tipo- } \\
\text { logías }\end{array}$ & V1 & V2 & V3 & V4 & V5 & V6 & V7 & V8 & V9 & V10 & V11 & V12 & V13 & V14 & V15 & $\begin{array}{c}\text { Puntaje } \\
\text { V16 com- } \\
\text { plejidad }\end{array}$ \\
\hline 1 & 1 & 4 & 4 & 4 & 1 & 4 & 4 & 1 & 4 & 4 & 4 & 4 & 1 & 1 & 4 & 4 & 49 \\
\hline 2 & 4 & 4 & 4 & 4 & 1 & 1 & 4 & 1 & 4 & 4 & 4 & 1 & 4 & 4 & 4 & 4 & 52 \\
\hline 3 & 4 & 4 & 4 & 1 & 1 & 4 & 1 & 1 & 4 & 4 & 4 & 1 & 4 & 1 & 4 & 4 & 46 \\
\hline 4 & 4 & 2 & 2 & 3 & 4 & 4 & 1 & 1 & 1 & 1 & 1 & 1 & 4 & 1 & 1 & 1 & 32 \\
\hline
\end{tabular}

Fuente: Elaboración propia.

A partir del resultado anterior, las tipologías obtenidas según complejidad son:

- Las tipologías 1 y 2, Complejidad extrema (49 y 52 puntos respectivamente);

- La tipología 3, Complejidad alta (46 puntos);

- La tipología 4, Complejidad media (32 puntos).

Las tipologías 1 y 2 al presentar similitud en sus variables de complejidad fueron agrupadas manteniendo el nombre de complejidad extrema, siendo graficado en la siguiente figura.

\section{TIPOLOGÍAS DE PERSONAS EN SITUACIÓN DE CALLE}

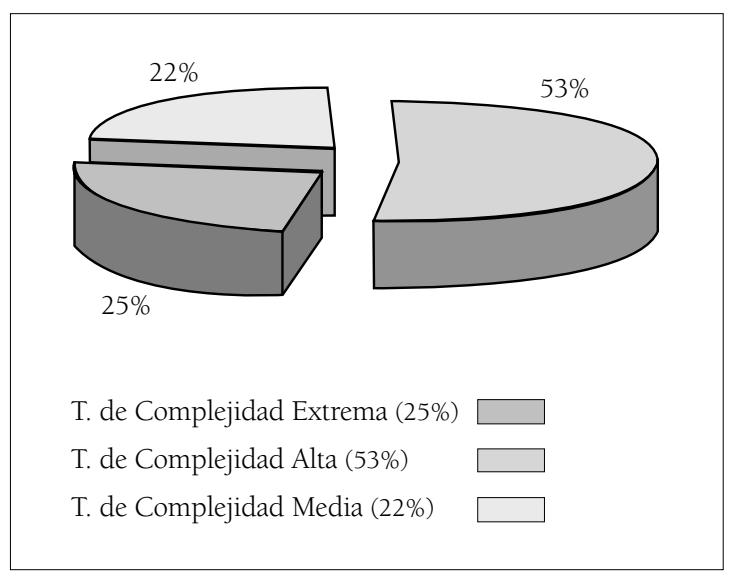

\section{Tipología de complejidad extrema}

Tipología que se encuentra conformada por el 53\% de la población en estudio, agrupando a un grupo significativo de mujeres. ${ }^{5}$ Este dato es relevante si se considera que la realidad en estudio es eminentemen- te masculina. Junto a ello, resulta ser la tipología más joven, conteniendo a los menores de 17 años, ${ }^{6} \mathrm{y}$ a los sujetos que se ubican en rangos de edad de 30 a 59 años. En su mayoría duermen en calle, situación que les transfiere mayor complejidad y vulnerabilidad, a diferencia de aquellos que pernoctan en hospederías, piezas u otros lugares. Tanto FEANTSA como MIDEPLAN concuerdan que vivir en situación de calle es la manifestación más seria de exclusión social, por resultar desestructurante (Bengoa \& Márquez, 1999; MIDEPLAN, 2005) y por adquirir rasgos de violencia, manifestado en la imposibilidad de satisfacer necesidades fundamentales, privacidad, afecto, bienestar físico, mental, trabajo (George,1994).

En cuanto al tiempo de permanencia en calle, es mayor a 5 años, y entre sus causas destacan problemas asociados al consumo problemático de drogas y alcohol. Además, en esta tipología se ubicarían sujetos con "rasgos de problemas psiquiátricos y cognitivos" (Rojas, 2007:6) desafiando mayormente las condiciones de la intervención. En cuanto al sistema laboral, en su mayoría declaran trabajar, la naturaleza de dichos trabajos es de carácter informal y cumplen una función instrumental y de corto plazo (Bravo, 2004:35). No cuentan con ingresos fijos, y tampoco transferencias estatales. Valoran negativamente su situación de calle, al considerarla riesgosa, y desesperanzadora.

5 La complejidad está dada, al reconocer que la situación de calle, "es una realidad eminentemente masculina, pues un 85\% de las personas en situación en calle son hombres." En, Ministerio de Planificación y Cooperación de Chile, División Social. (2005). Habitando la calle. Catastro nacional de personas en situación de calle. Santiago. Pág. 25.

6 Si bien este segmento es marginal cuantitativamente, se presenta desafiando a lógicas y equipos de trabajo tradicionales de intervención. Javier De Nicoló en Colombia logra abordar la complejidad de la situación de calle en que viven adolescentes desde lógicas renovadas, diferenciadas y flexibles. Véase. DE NICOLÓ, Javier. Musarañas. Bogotá. 1981. 


\section{Tipología de complejidad alta}

Tipología que representa el 25\% de la población en estudio, está conformada por un número menor de mujeres y es menos joven que la tipología anterior. Una parte de este grupo se caracteriza por dormir en calle, mientras que otra parte similar pernoctan en hospedería a pesar de señalar contar con vivienda. La permanencia en calle es menor que la tipología de complejidad extrema. En su mayoría señalan mantener contacto con la familia, y declaran no trabajar, ni machetear, como tampoco recibir transferencias estatales, situación que los mantiene excluidos del sistema económico, careciendo de reconocimiento positivo e intercambio material y simbólico(Op. Cit). Declaran vivir solos y presentar consumo problemático de drogas y alcohol al igual que la tipología de complejidad extrema. La situación de calle la asocian a aspectos negativos, principalmente riesgo y malas condiciones de vida.

\section{Tipología de complejidad media}

Tipología que representa el $22 \%$ de la población en estudio, concentra al $68,6 \%$ de personas mayores de 60 años. En su mayoría duermen en hospedería, siendo coincidentes con lo planteado por MIDEPLAN (2005) que señala que a mayor edad mayor inclusión en hospederías. Junto con residir en hospedería, dicen estar en situación de calle debido a problemas económicos. Su principal fuente de ingreso proviene de transferencias estatales. Estos ingresos resultarían insuficientes si pretendieran pensar en autonomía residencial. De lograrlo, los sujetos de esta tipología no cubrirían otras necesidades fundamentales. A su vez, declaran estar en calle por problemas de salud, por sobre otros (MINSAL, 2004). La situación de calle no representa aspectos negativos, a diferencia de las dos tipologías anteriores.
La ubicación de las tipologías de complejidad descritas, se encuentra dado por la relación complejidad/calidad. El resultado de dicha relación señala que a tipologías de complejidad extrema requiere de buenas condiciones de calidad. Recordemos que es la tipología que conglomera a los sujetos que presentan mayores exclusiones de los sistemas funcionales, por tanto, demandan condiciones sobre el umbral mínimo y medio.

En cuanto a las tipologías de complejidad alta, la matriz las ubica relacionada con condiciones de media calidad. Esta calidad esta en sintonía con las personas que se encuentran vinculadas a organizaciones del sistema de cooperación. Son aquellos que han incorporado o aceptado la propuesta de regulación desde las organizaciones, no obstante, demanda condiciones de intervención que potencien sustentabilidad, a objeto de evitar dependencia hacia las organizaciones.

En otro lugar de la matriz de análisis relacional complejidad/calidad, observamos a la tipología de complejidad media, la cual agrupa al mayor número de mujeres y adultos mayores, las condiciones de calidad del umbral mínimo apunta hacia la actualización de necesidades fundamentales junto con propiciar nuevos horizontes de inclusión.

Cada organización del sistema de cooperación que oriente su quehacer a la intervención social con personas en situación de calle, está llamada a resolver qué condiciones de calidad requiere implementar de acuerdo a la complejidad social en que se ubican los sujetos. Incorporar lógicas complejidad/calidad, responde a lógicas contemporáneas de abordar los fenómenos sociales, aportando al abandono de lógicas tradicionales de todos por igual.

\section{RESULTADO DEL ANÁLISIS RELACIONAL COMPLEJIDAD/CONDICIONES DE CALIDAD}

Matriz de análisis relacional complejidad/calidad

\begin{tabular}{|l|c|c|c|c|}
\hline \multicolumn{4}{|c|}{ Condiciones para intervenciones sociales de calidad con personas en situación de calle } \\
\hline \multirow{3}{*}{$\begin{array}{l}\text { Tipologías de } \\
\text { complejidad } \\
\text { de personas en } \\
\text { situación de calle }\end{array}$} & Buenas & \multicolumn{1}{|c|}{ Medias } & Umbral mínimo & Nivel insuficiente \\
\cline { 2 - 5 } & Extrema & $\begin{array}{l}\text { Tipologías de personas } \\
\text { en situación de calle de } \\
\text { complejidad extrema }\end{array}$ & & \\
\cline { 2 - 5 } & Alta & & $\begin{array}{l}\text { Tipologías de personas } \\
\text { en situación de calle de } \\
\text { complejidad alta }\end{array}$ & \\
\cline { 2 - 5 } & Media & & $\begin{array}{l}\text { Tipologías de personas } \\
\text { en situación de calle de } \\
\text { complejidad media }\end{array}$ \\
\hline
\end{tabular}

Fuente: Adaptación de "Matriz de análisis relacional de complejidad," presentada por Matus et.al. 2007. 


\section{Conclusiones y recomendaciones técnicas}

A partir de la diferenciación de la complejidad del fenómeno de las personas en situación de calle, se obtuvieron tres tipologías: complejidad extrema, complejidad alta, y complejidad media. No se encontraron tipologías de complejidad baja, reflejando el alto nivel de asociación de las variables que determinan exclusiones sociales en personas en situación de calle, junto con dar cuenta de la persistente complejidad del fenómeno que experimentan los sujetos. En cuanto a los sistemas funcionales, las principales conclusiones son:

- Sistema Familiar: Las tres tipologías dicen presentar contacto con la familia dentro del último año, siendo la tipología de complejidad alta, menos frecuente.

- Sistema Habitacional: La mayoría señala contar con vivienda, dando cuenta de lo multicausal del fenómeno, junto con disipar que la ausencia de vivienda sería la causa principal por la cual los sujetos dicen estar en calle. En relación a este sistema, las diferencias por tipología surgen según el lugar donde duermen. Los sujetos que señalan dormir en calle, se ubican en tipologías extremas y altas.

- Sistema Salud: Variable que marca distinciones por tipología, conglomerando en tipologías extrema y alta a sujetos que señalan presentar dependencias a sustancia y/o alcohol.

- Sistema Económico: Sólo la tipología de complejidad media, cuenta con ingresos fijos provenientes de transferencias estatales, situación que garantizaría en este nivel la inclusión a dicho sistema.

Al relacionar las tipologías encontradas con la matriz relacional complejidad/calidad, se concluye que las condiciones de calidad de la intervención varían según los niveles de complejidad, orientando condiciones de calidad de la intervención.

\section{Consideraciones técnicas: Tipología de complejidad extrema}

- Se requiere de buenas condiciones de calidad que consideren reconocimiento y vínculo con los sujetos para luego impulsar acciones de cambio.

- Elaborar diagnósticos que contemplen dimensiones familiares, habitacionales, económicas, profundizando, en el ámbito de la salud mental, al estar asociada a los motivos por los que dicen estar en calle: consumo problemático de drogas, y/o alcohol.

- Promover procesos de desintoxicación, compensación y/o reducción del daño, considerando la autodeterminación de los sujetos, identificando:

- Disposición a iniciar tratamiento: En este caso la intervención social buscará articular la inclusión al sistema terciario de salud.

- Sin disposición a iniciar tratamiento: En este caso la intervención social está llamada a intencionar condiciones de reducción del daño, donde motivar el ingreso a hospederías, es una de ellas.

- Articular la red de atención terciaria, y activar el ingreso a hogares protegidos como alternativa habitacional para aquellos sujetos que no resulte posible su inclusión al sistema familiar.

- Considerar mecanismos articuladores y de reestablecimiento de relaciones primarias y secundarias, siendo central en la adherencia y voluntariedad en el inicio de un tratamiento médico.

- Frente a sujetos que señalan no contar con redes familiares, o que teniéndolas no resulten funcionales, se sugiere fortalecer la figura tutorial que contempla el Plan Piloto de Integración Social planteado desde MIDEPLAN y/o el rol del voluntariado.

\section{Consideraciones técnicas: Tipología de complejidad alta}

- Se sugiere iguales consideraciones técnicas en cuanto al sistema de salud que la tipología anterior por presentar semejanzas.

- Modelos de intervención que no presionen el abandono de la situación de calle de manera repentina, al considerar que los sujetos han permanecido en dicha situación entre 1 y 5 años.

- Identificar qué solución habitacional requieren.

- Identificar aquellos sujetos que permanecen sostenidamente en hospedería a objeto de evaluar acciones que promuevan alternativas habitacionales permanentes.

- Contar con necesidades básicas de vivienda, alimentación y hábitos de higiene resueltas, para acceder al mercado laboral, siendo fundamental llegar a estos mínimos para iniciar procesos de incorporación y búsqueda de empleo. 
- Captar mediante la orientación sistémica contextual la inclusión a trabajos que ellos requieran y puedan realizar. Una intervención con sujetos de esta complejidad, no puede cometer errores al otorgar alternativas que no respondan a sus intereses y potencialidades.

\section{Consideraciones técnicas: Tipología de complejidad media}

- Indagar antecedentes familiares y/o redes significativas, no solo con el objeto de registro, sino para avanzar con ellos hacia la meta de inclusión, si ello responde a sus intereses.

- Propiciar el ingreso a programas residenciales distintos a hospedería y/o en arriendos autogestionados.

- En cuanto a su inclusión al sistema de salud, y considerando los problemas de enfermedades crónicas que caracteriza a este grupo, impulsar la inclusión a centros de atención primaria que favorezcan diagnósticos y tratamientos efectivos.

- Concursar a proyectos del Servicio Nacional del Adulto Mayor, SENAMA, propiciando espacios diurnos de integración que potencien autonomía y capacidades instrumentales.

\section{Recomendaciones finales:}

- Aplicar el modelo Complejidad/Calidad, en intervenciones sociales con personas en situación de calle. Aportando a la correspondencia entre las diversas exclusiones que experimentan los sujetos con las condiciones de calidad para transformarlas en inclusión.

- Diferenciar la complejidad para propiciar intervenciones de calidad. No es posible hablar de calidad de la intervención social si no existe correspondencia con la complejidad diferenciada.

- Incorporar lógicas de intervención de orientación sistémica contextual: La intervención de calidad no se impone, no hay coacción ni compulsión, son los sujetos los que aceptan y hacen suya la propuesta de intervención. Cuando esto ocurre, nos acercamos a políticas públicas de segunda generación que promueve autonomía y perspectiva de derechos. Lo anterior implica innovar, crear espacios diversos de intervención, dando saltos cualitativos en cuanto a las temáticas abordadas y los horarios para hacerlo. Crear espacios diurnos de acceso a la cultura, de uso de espacios públicos, de apresto laboral, de arte, de promoción de derechos.

- Equipos de trabajo: Se considera que a mayor complejidad de la intervención, mayor y mejores profesionales en su abordaje. La realidad requiere equipos de trabajo especializado y multidisciplinario. Es fundamental abandonar lógicas de intervención, únicamente desde lo social.

- Fortalecer el rol del voluntariado que trabaja con personas de situación de calle, impulsando la figura de "voluntario asistente," procurando su capacitación, a fin de dotarlos de una mirada integral del fenómeno, y de mejores herramientas en su rol de acompañamiento.

- Optimizar recursos provenientes del sistema de Protección Chile Solidario del cual forman parte las persona en situación de calle e identificar los beneficios estatales existentes optimizando su uso.

- Acoplamiento estructural, con programas del entorno: Acoplarse estructuralmente aquellos sistemas responsables de atender a personas en situación de calle que se transformen en obstáculos al desarrollo de los sujetos. Junto a ello, impulsar transformar las estigmatizaciones negativas en reconocimiento positivo de quienes tienen la calle por hogar. Acoplarse, a su vez, al sistema estatal, cada vez, que los derechos sociales de las personas en situación de calle sean vulnerados.

- Sustentabilidad de la intervención: Intencionar que personas en situación de calle al lograr inclusión logren transmitirla mediante acciones similares a otros que vivencien situaciones de exclusión. Con ello, se aporta a la sustentabilidad de la intervención recibida, acortando la dependencia hacia las organizaciones.

- Considerar la variable género en los objetivos de intervención, variable desdibujada frente a una realidad eminentemente masculina y con ello propiciar futuras investigaciones que recojan esta complejidad de la realidad.

- Ajustes permanentes, aportando en mejora continúa. Esta mejora continúa, implica identificar aquellas personas en situación de calle que no aparecen en las cifras, como tampoco en los registros. Llegar donde otros no llegan, es también un desafío de una intervención de calidad. 
- Reeditar, la experiencia del catastro nacional, profundizando en variables de salud mental, género, familia, redes de apoyo. En la misma línea de la investigación, se hace necesario, contar con estudios respecto al impacto de las intervenciones sociales en personas en situación de calle, desde que han sido incorporadas al Sistema de Protección Social Chile Solidario.

\section{Bibliografía}

BARROS, P., DE LOS RÍOS, D., \& TORCHE, F. (1996). "Lecturas sobre la exclusión social." Oficina Internacional del trabajo, OIT. Santiago.

BENGOA, J. \& MÁRQUEZ, F. (1999). "La desigualdad." Ediciones Sur. Santiago, Chile.

BRAVO. R. (2004). "Personas en situación de calle y trabajo." Tesis para optar al grado de licenciado en psicología. Universidad Diego Portales, Santiago, Chile.

CARITAS, ESPAÑOLA. (2006). "Sin techo sin derechos. Hoy también duermo en la calle. A la sociedad le falto yo." Madrid, España.

CEA D' ANCONA, MARÍA ÁNGELES. (2002). "Análisis Multivariable. Teoría y Práctica en la investigación Social." Editorial Síntesis. Madrid.

CHILE. MINISTERIO DE PLANIFICACIÓN Y COOPERACIÓN. "Ley 19.949. Sistema de Protección Social Chile Solidario." Julio 2006. En <http://www. bcn.cl/lc/bleyes>. Visitada 6 Noviembre de 2007.

DE NICOLÓ, JAVIER. (1981) Musarañas. Bogotá.

EUROPE FEDERATION OF NATIONAL ORGANIZATIONS WORKING WITH DE HOMELESS, FEANTSA (2001), "La promoción de la inclusión social a través del acceso al alojamiento, Documento Político."

EUROPE FEDERATION OF NATIONAL ORGANIZATIONS WORKING WITH DE HOMELESS, FEANTSA (2006). El Derecho a la Salud es un Derecho Humano: Garantizar la salud sanitaria a las personas que son sin-techo.

EUROPE FEDERATION OF NATIONAL ORGANIZATIONS WORKING WITH DE HOMELESS, FEANTSA (2007). Múltiples Barreras Soluciones Múltiples: la inserción en y a través del empleo de las personas sin hogar en Europa.

GEORGE, SUSAN. (2007) "Violencia y pobreza: Una relación estrecha." En papeles No 50, 1994. En www. fuhem.es. Visitada 20 de Octubre de 2007.

GERBER, M. (2006). Complejidad: Teoría y Método. Memoria para optar al grado de Socióloga. Universidad de Chile. Facultad de Ciencias Sociales. Departamento de Sociología. Santiago, Chile.
GÓMEZ, MÁXIMO. (1999). "Los derechos humanos." Documentos básicos. Editorial Jurídica de Chile. Santiago.

HERNANDEZ, SAMPIERI Y FERNÁNDEZ, CARLOS. (2000). Metodología de la Investigación. Editorial Mc GrawHill. México.

LUHMANN, NIKLAS. (1992). "Sociedad y Sistema: La Ambición de la Teoría." Editorial Paidos, Barcelona.

LUHMANN, NIKLAS. (1998). "Complejidad y Modernidad. De la unidad a la diferencia." Editorial Trotta.

MASCAREÑO, ALDO. (1996). Intervención en sistemas sociales autónomos. Tesis de Magíster. Santiago: Instituto de Sociología, Universidad Católica de Chile.

MASCAREÑO, ALDO. (2005). "Cuando un individuo importa". Presentado en Seminario el pueblo de la calle. Universidad Alberto Hurtado. Santiago. Chile.

MASCAREÑO, ALDO. (2006). "Sociología de la Solidaridad. La diferenciación de un sistema global de cooperación". Ediciones Universidad de Chile. Santiago.

MASCAREÑO, ALDO. (2007). "Sociología de la Intervención: Orientación Sistémica Contextual. En Adriana Kaulino (Ed), Mediación y complejidad, Universidad Diego Portales, Santiago.

MATUS, TERESA. (1999). "Propuestas Contemporáneas en Trabajo Social. Hacia una intervención polifónica". Espacio Editorial. Buenos Aires.

MATUS, TERESA. (2004). "El peso que queda. Condiciones de efectividad en los programas de acción social." EXPANSIVA, Santiago.

MATUS, TERESA. (2007). "Innovación e Intervención social." Ponencia presentada en Centro de Estudios de Emprendimiento Solidarios, CEES. Pontificia Universidad Católica. Santiago, Chile.

MATUS, T., IBARRA, R., FUENTES, L. \& PÉREZ U. (2007). La Reforma Municipal en la mira. Identificado los municipios prioritarios en la Región Metropolitana: Complejidad comunal versus condiciones para la gestión municipal. Expansiva/ Pontificia Universidad Católica. Santiago.

MEERT, H., MAUREL, E., WOLF, J., SARALA N., MAAS R., KOCH-NIELSEN I., CHRISTENSEN I. \& CABRERA P. (2005). "The Changing profiles of homelees people: still depending on emergency -services in Europa: Who an Why". Federation of National Organizations Working with de Homeless. FEANTSA.

MINISTERIO DE PLANIFICACIÓN Y COOPERACIÓN DE CHILE, DIVISIÓN SOCIAL, MIDEPLAN (2005). "Habitando la Calle. Catastro nacional de personas en situación de calle". Santiago - Chile. 
MINISTERIO DE PLANIFICACIÓN Y COOPERACIÓN DE CHILE, DIVISIÓN SOCIAL, MIDEPLAN (Secretaría ejecutiva Chile Solidario, (2006). Programa de Apoyo a la Integración Social de Personas en Situación de Calle. Estrategia de Intervención en la fase piloto del programa. Santiago - Chile.

MINISTERIO DE PLANIFICACIÓN Y COOPERACIÓN DE CHILE, DIVISIÓN SOCIAL, MIDEPLAN (2007). "Listado de Instituciones ejecutoras Programa de apoyo a la Integración social de personas en situación de calle. Estrategia de intervención fase piloto del programa". Santiago - Chile.

MINISTERIO DE PLANIFICACIÓN Y COOPERACIÓN DE CHILE, DIVISIÓN SOCIAL, MIDEPLAN (2007). "El contexto del programa calle y lógica de su operación." Taller de trabajo, coordinadores de proyecto. Santiago, 3 de Abril.

MINISTERIO DE SALUD. (2004). Encuesta Nacional de Salud. Santiago, Chile.

O' CONNELL, JAMES. (2004). The Health Care of Homelees Persons. Manual of communicable Diseases Common Problems in Shelters on de Steets. Boston.
OSSA, L. (2005). "Adolescente en situación de calle: construcción de identidad en situación de extrema vulnerabilidad." Tesis para optar al grado de Magíster. Universidad de Chile, Santiago, Chile.

RAZETO, LUIS. (1993). "Los caminos de la Economía Solidaria.” Editorial Vivarium. Santiago.

RIQUELME, VERÓNICA. (2005). Chile Solidario y los desafíos de la igualdad. Oficina Regional de la OIT para América Latina y el Caribe. Santiago. Chile.

RODRÍGUEZ, D \& RÍOS, RENÉ. (2007). "Las organizaciones sociales en una sociedad compleja." Perspectivas/2. Centro de estudios de emprendimiento solidarios. CEES. Pontificia Universidad Católica de Chile.

ROJAS, NICOLÁS. (2007). Más allá de las carencias. Tipologías de Personas en Situación de Calle. Fundación Hogar de Cristo. Área de estudios. Proyecto Promueve. Santiago, Chile.

SEN, AMARTYA. (1995). "Nuevo Examen de la desigualdad." Alianza Editorial. Madrid.

VICENTE, TERESA. (2006). "La exigibilidad de los derechos sociales." Editorial Tirant Lo Blanch. Valencia. 